\title{
NEUTRINO ASTRONOMY WITH ICECUBE
}

\author{
TYCE DeYOUNG \\ Department of Physics, Pennsylvania State University, 104 Davey Laboratory, \\ University Park, PA 16802, USA \\ deyoung@phys.psu.edu
}

Received 24 June 2009

\begin{abstract}
IceCube is a kilometer-scale high energy neutrino telescope under construction at the South Pole, a second-generation instrument expanding the capabilities of the AMANDA telescope. The scientific portfolio of IceCube includes the detection of neutrinos from astrophysical objects such as the sources of the cosmic rays, the search for dark matter, and fundamental physics using a very large data set of atmospheric neutrinos. The design and status of IceCube are briefly reviewed, followed by a summary of results to date from AMANDA and initial IceCube results from the 2007 run, with 22 of a planned 86 strings operational. The new infill array known as Deep Core, which will extend IceCube's capabilities to energies as low as $10 \mathrm{GeV}$, is also described.
\end{abstract}

Keywords: IceCube; neutrinos; dark matter.

PACS Nos.: 95.55.Vj, 95.85.Ry, 95.35.+d

\section{Cosmic Rays, Gamma Rays, and Neutrinos}

The origin of the cosmic rays has been the subject of intense study since their discovery by Victor Hess in 1912. Today we know that cosmic sources accelerate particles to energies above $10^{20} \mathrm{eV}$, although we are uncertain about the dynamics or indeed the identities of those sources. The spectrum of the cosmic rays follows a broken power law; those below a break at approximately $3 \mathrm{PeV}$ known as the "knee" are believed to originate from sources in our Galaxy. Extragalactic origins are inferred for cosmic rays at the highest energies, as no Galactic objects are believed powerful enough to accelerate particles to those energies. The precise energy scale of the transition remains a matter of some debate. ${ }^{1,2}$

The energetic environments in which high energy cosmic rays are accelerated are likely to include matter or radiation fields with which the accelerated hadrons will interact, producing charged pions or kaons which decay to neutrinos. ${ }^{\text {a }}$ The same processes will also produce neutral pions, which will decay into gamma rays. The

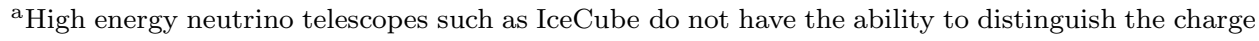
of observed particles, so we will not distinguish between particles and antiparticles in this review. 
energies of the gamma rays will be comparable to those of the neutrinos, ${ }^{3}$ so sources of very high energy (VHE, used herein to indicate energies of roughly $100 \mathrm{GeV} \sim$ $1 \mathrm{PeV}$ ) neutrinos would also be candidates for VHE gamma ray emission, and vice versa. However, $\mathrm{TeV}$ gamma rays may also be produced by accelerated electrons inverse-Compton scattering lower energy photons. Neutrinos would not be produced in this case, so neutrinos provide an unambiguous indicator of hadronic acceleration. Conversely, neutrino emitters may be "hidden" if their local environments are too dense for the gamma rays to escape, ${ }^{4}$ or may only be visible at lower $(\sim 10 \mathrm{GeV})$ energies if the sources are sufficiently distant that $\mathrm{TeV}$ gammas are attenuated by interaction with the extragalactic background light. ${ }^{5}$

Neutrino production through $\pi / K$ decay leads generically to a neutrino flavor ratio of $\nu_{e}: \nu_{\mu}: \nu_{\tau}=1: 2: 0$ at the source. However, for essentially all astrophysically relevant distances and energies, the neutrinos will oscillate on their way to Earth to an equilibrium flavor ratio of $1: 1: 1$. This prediction of the flavor ratio is not absolute, however, and deviations can carry information about astrophysical neutrino sources. ${ }^{6-9}$ Very extreme flavor ratios could be indicators of new fundamental physics, such as neutrino decay. ${ }^{10}$

Neutrinos are detected by IceCube when they undergo either charged current (CC) or neutral current (NC) interactions with nucleons in the ice. At VHE energies, these interactions are deeply inelastic, producing a hadronic shower of relativistic particles. In CC interactions, a secondary lepton corresponding to the flavor of the incident neutrino $(e, \mu$, or $\tau)$ is also produced, carrying between one half and threequarters of the neutrino energy ${ }^{20}$ and aligned with the neutrino direction to better than a degree at energies above the TeV scale. ${ }^{21}$

Muons thus produced may travel through the ice for kilometers in a nearly straight trajectory, depending on their energy. They are detected as long, straight tracks in the detector via the Cherenkov radiation they emit. At higher energies, stochastic interactions (bremsstrahlung etc.) produce small showers that are not resolved individually but which increase the apparent brightness of the track. Electrons produce a compact $(\sim 10 \mathrm{~m})$ electromagnetic shower intermingled with the hadronic shower at the vertex, from which Cherenkov radiation propagates out in a roughly spherical pattern. At low energies, tau leptons are indistinguishable from electron cascades due to the rapid $\tau$ decay. However, at higher energies, above the $\mathrm{PeV}$ scale, a long tau track can be observed, usually with a cascade on either end due to the initial neutrino-nucleon interaction and the final tau decay. ${ }^{22}$ Events in which only one cascade is observed within the detector ${ }^{10}$ or in which the $\tau$ decays to $\mu$ inside the detector ${ }^{23}$ may also be identifiable.

Large-volume neutrino telescopes such as IceCube can also be used to search for other particles that would produce light in the detector, such as magnetic monopoles, long-lived supersymmetric particles, ${ }^{24}$ and other exotica. Searches for such particles are in progress but are beyond the scope of this review. 
Neutrino detection is complicated by the background of atmospheric muons produced via meson decay in cosmic ray air showers. Neutrino telescopes are sited at least a kilometer below the surface to reduce this muon flux, but the high-energy tail of the flux penetrating to these depths is still many orders of magnitude more numerous than the VHE neutrino fluxes of interest. This background is controlled by searching for upward-going muons, which must have been produced in neutrino interactions below the detector. Other event signatures characteristic of neutrinos, such as isolated cascades within the detector or downgoing muon tracks starting from a neutrino interaction vertex observed within the detector, can also be used.

Potential sources of VHE neutrinos include Galactic sources such as supernova remnants and extragalactic sources such as active galactic nuclei and gamma ray bursts; several recent reviews ${ }^{11-13}$ discuss these sources in detail. Independent of the identities of the sources, the observed flux of cosmic radiation allows us to make an estimate of the total number of neutrinos produced by hadrons accelerated in the universe, ${ }^{14}$ albeit one somewhat dependent on assumptions about the opacity and distance distribution of these sources. ${ }^{15}$ The coming generation of kilometer-scale neutrino telescopes, such as IceCube and $\mathrm{KM} 3 \mathrm{NeT},{ }^{16}$ will be sensitive to fluxes significantly below this level, suggesting that the prospects for detection of VHE neutrino sources in the near future are good.

\section{The IceCube Detector}

IceCube consists of a three-dimensional array of photodetectors known as Digital Optical Modules (DOMs), buried at depths of $1450 \mathrm{~m}$ to $2450 \mathrm{~m}$ in the Antarctic ice cap near the South Pole. The DOMs are mounted on vertical cables (called "strings") of 60 DOMs separated by $17 \mathrm{~m}$. Eighty such strings will be deployed on a triangular grid with a horizontal spacing of $125 \mathrm{~m}$ between strings, covering a surface area of approximately $1 \mathrm{~km}^{2}$. As of the end of the 2008-09 austral summer construction season, a total of 59 strings have been deployed, as shown in Fig. 1. Completion of the full IceCube detector is expected in February 2011.

Each DOM houses a $25 \mathrm{~cm}$ Hamamatsu photomultiplier tube (PMT) and associated electronics within a glass pressure vessel. The onboard electronics record the PMT signals at a sampling rate of $300 \mathrm{MHz}$ in the first $400 \mathrm{~ns}$ (a slower fADC records late light at a rate of $40 \mathrm{MHz}$ ) with an instantaneous dynamic range of approximately 1,000 photoelectrons per $10 \mathrm{~ns}$. The absolute timing accuracy of each DOM is better than $3 \mathrm{~ns}$. The DOMs communicate asynchronously to a softwarebased data acquisition (DAQ) system on the surface, which forms triggers and builds events. ${ }^{17}$

IceCube also includes several specialized subdetectors. An array of (frozen) water tanks on the surface, known as IceTop, ${ }^{18}$ is sensitive to extensive air showers produced by cosmic rays in the atmosphere. There is one IceTop station, consisting of two tanks with a diameter of $2 \mathrm{~m}$ and an ice depth of $90 \mathrm{~cm}$, at the top of each IceCube string. Each tank contains two DOMs, operating at different gains to 


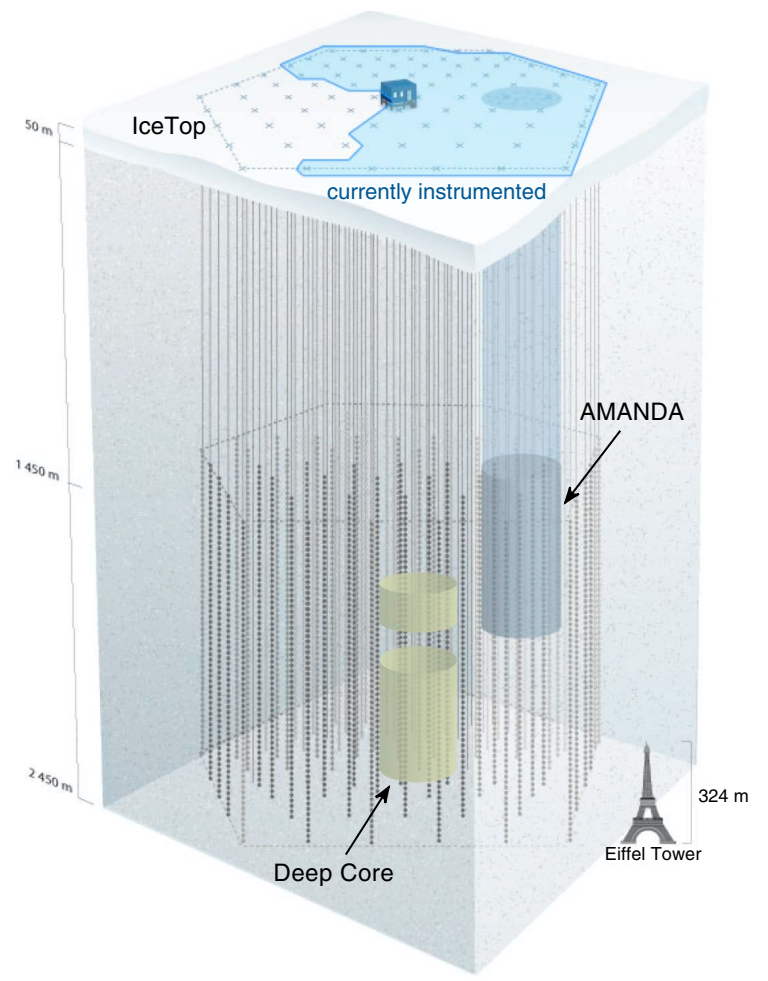

Fig. 1. Schematic of the IceCube detector. Each black dot represents one DOM. The surface area corresponding to the strings and IceTop stations deployed as of 2009 is shaded blue, and the volumes occupied by the AMANDA and Deep Core subarrays are indicated. The Eiffel Tower is shown for scale.

further extend the dynamic range. IceTop will be used in conjunction with IceCube to study the composition of cosmic rays above the "knee" in the spectrum around $3 \mathrm{PeV}$. It can also be used for solar physics, as for example with the measurement of the particle spectra associated with the solar event of December 13, 2006. ${ }^{19}$

The first-generation South Pole neutrino telescope, AMANDA, is colocated with IceCube. Following seven years of operation in its final configuration, AMANDA was incorporated into IceCube and operated as a subdetector for two years until being decommissioned in May 2009. The much denser spacing of the AMANDA OMs led to a lower energy threshold for events in the jointly instrumented region, but its dissimilar instrumentation rendered joint operations complex, and its location near the top edge of IceCube made background rejection difficult.

\subsection{Deep Core}

The IceCube collaboration recently decided to expand the capabilities of the detector at lower energies with the addition of Deep Core, an infill array that will be located at the bottom center of IceCube, as shown in Fig. 1. Deep Core will comprise 
the seven innermost standard IceCube strings, as well as six new strings deployed in a ring of radius $72 \mathrm{~m}$ around the central string. The six new strings will each mount 60 DOMs, 50 of which will be deployed on a $7 \mathrm{~m}$ spacing between $2100 \mathrm{~m}$ and $2450 \mathrm{~m}$ below the surface. The remaining 10 DOMs will be deployed at shallower depths to improve the efficiency of detection of extremely vertical background muons. With a radius of $125 \mathrm{~m}$ and a height of $350 \mathrm{~m}$, the instrumented volume of Deep Core will include 15 Mton of ice, with expected sensitivity to neutrinos at energies as low as $\sim 10 \mathrm{GeV}$.

The new DOMs will be identical to standard IceCube DOMs except that they will use a new model of PMT developed by Hamamatsu to increase the quantum efficiency of the photocathode. Lab tests with assembled DOMs indicate the sensitivity of the high-QE PMTs is approximately $30 \%$ higher than that of standard DOMs. The denser DOM spacing and higher DOM sensitivity combined will increase the collection of photons in the Deep Core volume by approximately a factor of 10. Furthermore, the ice at Deep Core depths is significantly more transparent than that at shallower depths, with optical attenuation lengths of 40-45 m compared to $20-25 \mathrm{~m}$ in the top of the detector. The significantly improved light collection in Deep Core translates to much higher sensitivity to relatively dim, low energy neutrino events. Additionally, the bulk of IceCube can be used to detect and veto atmospheric muons penetrating to Deep Core. The ratio of the atmospheric muon trigger rate to the atmospheric neutrino trigger rate in IceCube is approximately $10^{6}$; initial Monte Carlo studies indicate that veto efficiencies on this order are achievable with relatively good signal efficiency.

The first of the six new Deep Core strings was successfully deployed at South Pole in the 2008-09 austral summer. Deployment of the remaining five new strings, as well as the standard strings that compose the Deep Core array, is scheduled to be complete by February 2010.

\section{Atmospheric Neutrinos}

The flux of atmospheric neutrinos produced in cosmic ray air showers in the Earth's atmosphere constitutes a background to searches for astrophysical neutrinos. They can be distinguished by their much softer spectrum, $d N / d E_{\nu} \sim E_{\nu}^{-3.7}$ at $\mathrm{TeV}$ energies $^{25}$ instead of the approximately $E_{\nu}^{-(2+\delta)}$ generally expected from shockaccelerated hadronic production. ${ }^{26}$ Also, the flavor ratio $\nu_{e}: \nu_{\mu}: \nu_{\tau}$ is approximately $0.05: 1: 0$ at $\mathrm{TeV}$ energies, ${ }^{26}$ as opposed to the approximate flavor equality expected from astrophysical sources. At higher energies, "prompt" neutrino production in the decay of short-lived charmed mesons becomes important, leading to a harder spectrum and a flavor ratio tending to $1: 1: 0.1{ }^{27-29}$ Relatively weak limits have been placed on the magnitude of the prompt $\nu_{\mu}$ flux with AMANDA ${ }^{30}$ and better measurements will be possible with IceCube.

While atmospheric neutrinos constitute a background to astrophysical neutrinos, their existence also provides a useful tool both for understanding the response 

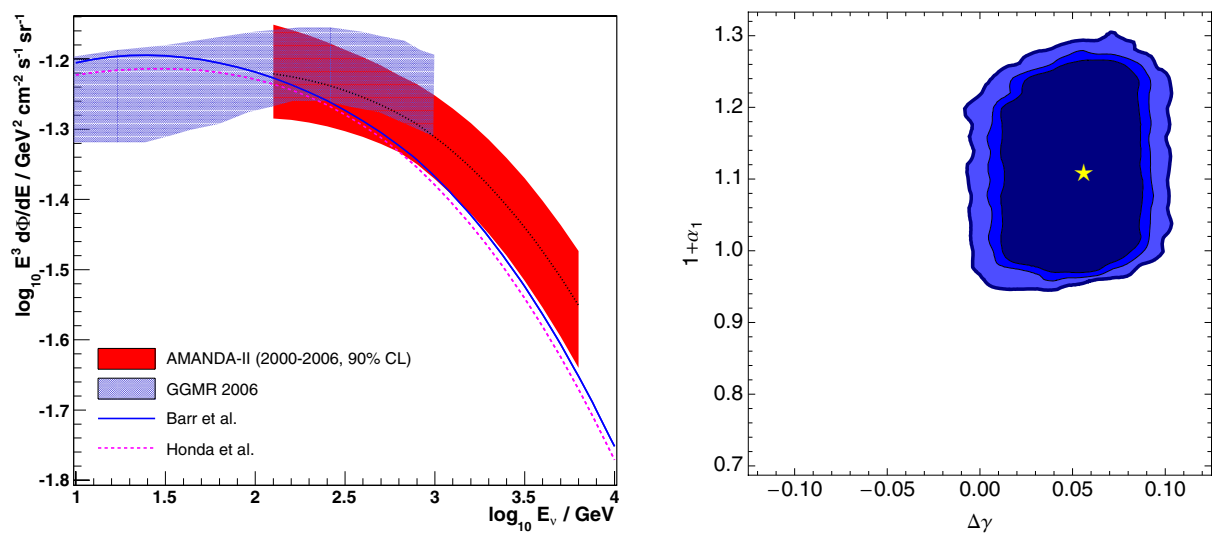

Fig. 2. The atmospheric $\nu_{\mu}+\bar{\nu}_{\mu}$ flux observed by AMANDA-II. The $90 \%, 95 \%$ and $99 \%$ C.L. contours and best fit point in relative flux normalization and spectral index $(\Delta \gamma)$ of the flux, relative to the Bartol prediction, are shown at right.

of the IceCube detector and for particle physics. The atmospheric flux is reasonably well known in the VHE energy range, with a precision of about $30 \%$ in the muon neutrino rate at $1 \mathrm{TeV} .{ }^{31}$ Two independent calculations of the atmospheric muon neutrino flux ${ }^{32,33}$ are shown in Fig. 2; note that the deviation between the two calculations is not a good indicator of the total uncertainty, because the latter is dominated by uncertainties in external factors used as inputs by both calculations, such as charged kaon production at high energies.

A measurement of the atmospheric $\nu_{\mu}+\bar{\nu}_{\mu}$ flux using the full AMANDA-II data set $^{34}$ is shown in Fig. 2, along with a measurement extracted from the published Super-Kamiokande data set ${ }^{35}$ by Gonzalez-Garcia et al. ${ }^{36}$ The results begin to suggest a flux slightly (10\%) higher and harder (shift in spectral index $\Delta \gamma \sim 0.06$ ) than the central value calculated by Barr et al., but the current AMANDA results are consistent with the central value at the $90 \%$ to $99 \%$ C.L. in both parameters. IceCube will rapidly produce a much larger data set, so improvements may be expected in the near future: the quarter-built IceCube detector observed roughly as many atmospheric neutrinos in one year of operation as AMANDA did in seven.

The large number of atmospheric neutrinos detected by AMANDA can be used to search for evidence of physics beyond the Standard Model, such as quantum decoherence or violations of Lorentz invariance. ${ }^{34}$ These phenomena would appear as oscillation-like behavior, but at higher energies than standard neutrino oscillations and with different characteristic flavor signatures. The reach of such studies will be greatly improved in the future with the completion of IceCube and the addition of Deep Core: IceCube will collect a sample of well over a million atmospheric neutrino events, with energies ranging from $\sim 10 \mathrm{GeV}$ up to hundreds of $\mathrm{TeV}$. In addition to extending the analyses mentioned above, such a large neutrino data set should permit observation of neutrino oscillations, including tau appearance. Furthermore, if the $\theta_{13}$ mixing angle is large (near present limits), a determination 
of the neutrino mass hierarchy might be possible, albeit quite difficult, by exploiting matter-enhanced mixing though the Earth along with asymmetries between the neutrino and antineutrino interaction cross sections and inelasticity distributions ${ }^{37}$ at energies around $10 \mathrm{GeV}$.

\section{Astrophysical Neutrinos}

Searches for individual astrophysical sources of neutrinos have been conducted separately on both the complete seven year AMANDA-II data and the one-year data set collected by the 22 IceCube strings operational in 2007. Analysis of more recent IceCube data is underway, and will lead to significant increases in sensitivity.

The AMANDA-II search uses a sky map of 6,595 upward going neutrino events collected between 2000 and $2006 .^{38}$ The direction of each of these events was reconstructed using a maximum likelihood method accounting for the propagation of light in the ice, ${ }^{39}$ with the width of the solution in the likelihood space used to estimate the angular resolution for each event and the number of OMs detecting light serving as an energy estimator. A list of 26 energetic galactic and extragalactic objects was defined a priori as candidate neutrino sources, including active galactic nuclei, supernova remnants, and $\mathrm{TeV}$ gamma ray sources identified by Milagro in the Cygnus region ${ }^{40}$ (now identified with $\mathrm{GeV}$ pulsars or pulsar wind nebulae ${ }^{41}$ on the basis of correlations with the Fermi Bright Source List ${ }^{42}$ ). At the location of each candidate, a likelihood ratio was calculated comparing the hypothesis that all nearby neutrinos were atmospheric to a hypothesis also including a neutrino source contributing $\hat{n}_{s}$ signal events with a spectral index $\hat{\gamma}$. The significance ( $p$-value) of the likelihood ratio was assessed by repeatedly randomizing the right ascension of each event in the data set to create pure background maps that reproduce any zenith-dependent asymmetries in the detector response, but with any real neutrino sources smeared out across the sky.

A similar search was carried out using the 2007 IceCube 22 -string data set. ${ }^{43}$ This set, collected from May 2007 to April 2008, contains 5,114 neutrino candidate events detected in 276 days of live time, consistent with an expectation of $4600 \pm$ 1400 atmospheric neutrino events and $400 \pm 200$ cosmic ray muons misreconstructed as upgoing neutrino events. The source list was expanded in this search to include 28 candidate sources, most of which were identical to the candidates in the AMANDAII list. Results for selected sources are shown in Table 1, including those with the smallest $p$-values in each search. In neither case are these $p$-values inconsistent with the background hypothesis: one expects to obtain $p \leq 0.0086$ for at least one of 26 sources in $20 \%$ of signal-free sky maps, and $p \leq 0.071$ in $66 \%$. The $90 \%$ C.L. upper limits placed on $\nu_{\mu}$ emission from the sources, assuming $E^{-2}$ spectra and $1: 1: 1$ flavor ratios, are also shown in Table 1.

In addition, unbinned all-sky searches were conducted using both data sets, testing points on $0.25^{\circ} \times 0.25^{\circ}$ grids from declination $-5^{\circ}$ to $83^{\circ}$ for the AMANDAII data set and to $85^{\circ}$ for the IceCube-22 data set. (Higher declinations will be used 
Table 1. Selected $p$-values and $90 \%$ C.L. upper limits on $\nu_{\mu}+\bar{\nu}_{\mu}$ fluxes $E_{\nu}^{2} d N / d E \leq \Phi_{90} \times$ $10^{-12} \mathrm{TeV} / \mathrm{cm}^{2} \mathrm{~s}$, from searches for neutrino emission from predefined candidate sources with AMANDA and IceCube-22. Dashes indicate the best-fit number of signal events was zero.

\begin{tabular}{lccccrr}
\hline \multicolumn{1}{c}{ Source } & decl. $\left[^{\circ}\right]$ & r.a. $[\mathrm{h}]$ & AMANDA $p$-value & IC22 $p$-value & $\Phi_{90}^{\mathrm{AM}}$ & $\Phi_{90}^{\mathrm{IC} 22}$ \\
\hline Crab Nebula & 22.01 & 5.58 & 0.10 & - & 46.4 & 10.35 \\
Geminga & 17.77 & 6.57 & 0.0086 & - & 63.9 & 9.67 \\
MGRO J2019+37 & 36.83 & 20.32 & 0.077 & 0.25 & 48.4 & 25.23 \\
LS I +61 303 & 61.23 & 2.68 & 0.034 & - & 73.7 & 22.00 \\
XTE J1118+480 & 48.04 & 11.30 & 0.50 & 0.082 & 25.9 & 40.62 \\
Cygnus X-1 & 35.20 & 19.97 & 0.57 & - & 20.0 & 14.60 \\
Mrk 421 & 38.21 & 11.07 & 0.82 & - & 12.7 & 14.35 \\
Mrk 501 & 39.76 & 16.90 & 0.22 & 0.071 & 36.4 & 14.44 \\
1ES 1959+650 & 65.15 & 20.00 & 0.44 & - & 22.5 & 59.00 \\
M87 & 12.39 & 12.51 & 0.43 & & 7.91 \\
\hline
\end{tabular}

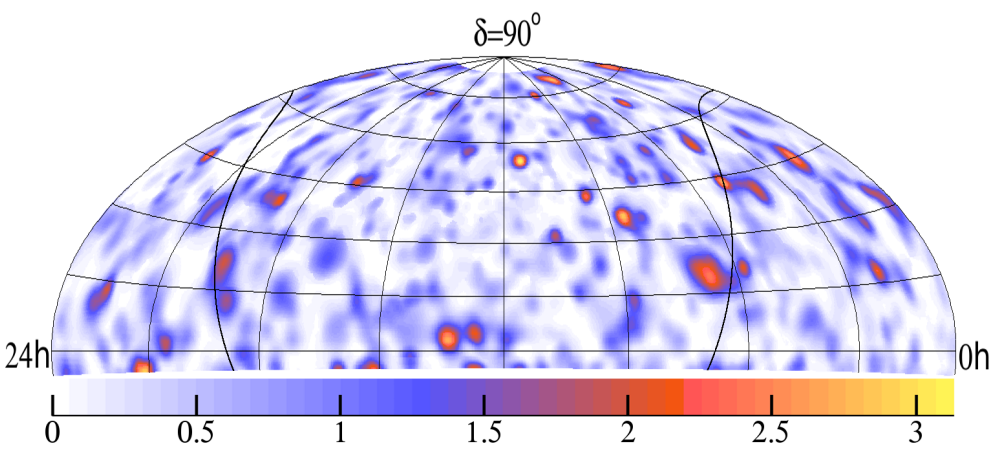

Fig. 3. Map of the pre-trials significances (in $\sigma$ ) obtained from an unbinned point source search using the full AMANDA-II data set. The most significant point has a significance of $3.38 \sigma ; 95 \%$ of randomized background skymaps include a point at least as significant.

to search for dark matter in the Earth's core.) The results of the AMANDA-II search are shown in Fig. 3. The most significant point on the sky, at $\delta=54^{\circ}$ and $\alpha=11.4 \mathrm{hr}$, had a significance of $3.38 \sigma$ before accounting for the large number of points tested. In $95 \%$ of randomized maps, a point with at least $3.38 \sigma$ was found, indicating that such a level is consistent with statistical fluctuations of the background.

The results of the all-sky search using IceCube data are shown in Fig. 4. The most significant deviation from the background expectation, with $\hat{n}_{s}=7.7$ events, occurred at $153.4^{\circ}$ right ascension, $11.4^{\circ}$ declination. The pre-trials significance of this deviation is estimated to be $7 \times 10^{-7}$, but more significant deviations were observed in 67 out of 10,000 scrambled background maps. Accounting for the parallel search for emission from sources selected a priori also performed on the data set, as described above, the final $p$-value of the result is estimated to be $1.34 \%$. This is not sufficient to reject the background-only null hypothesis. 


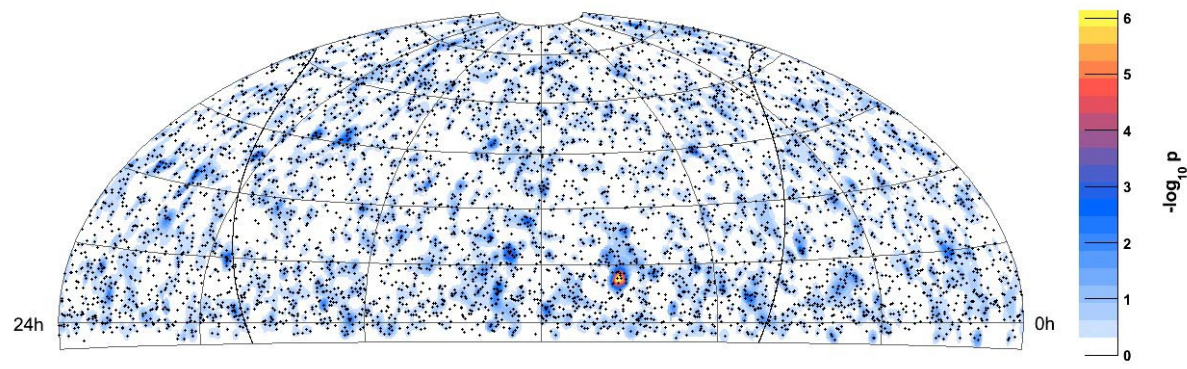

Fig. 4. Map of the pre-trials $p$-values obtained from an unbinned point source search using the 22-string 2007 IceCube data set. Dots indicate the individual events used to calculate the significances. The probability of observing in signal-free maps a point at least as significant as the brightest spot in this map is estimated to be $1.34 \%$, insufficient to reject the background hypothesis.

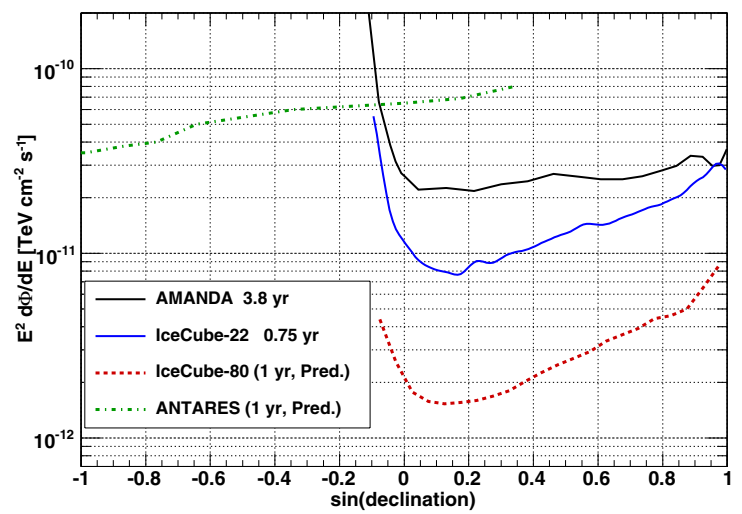

Fig. 5. Average $90 \%$ C.L. upper limit on the $\nu_{\mu}+\bar{\nu}_{\mu}$ flux (assuming flavor equality) from a point source as a function of declination, compared to predictions for IceCube and ANTARES.

The sensitivities of the two all-sky searches are compared in Fig. 5, for $\nu_{\mu}$ fluxes $d N / d E \sim E^{-2}$. Predicted sensitivities for one year of operation with the full IceCube and with ANTARES ${ }^{44}$ are also shown. One year of operation with only one quarter of the full IceCube array already provides a sensitivity significantly surpassing that of the seven-year AMANDA-II data set. This sensitivity will increase rapidly as IceCube construction were completed, so if the "hot spot" seen in the IC22 sky map is indicative of a neutrino source rather than simply a background fluctuation, the source would be definitively detected in the very near future.

\subsection{Gamma ray bursts}

Searches for neutrino emission in coincidence with gamma ray bursts (GRBs) observed via their electromagnetic emission have also been conducted, with both AMANDA and IceCube. These analyses benefit from drastically lower background rates, due to the external information regarding the time and direction of the bursts. 


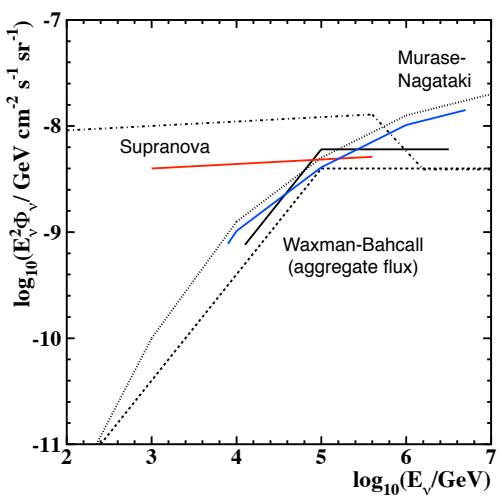

(a)

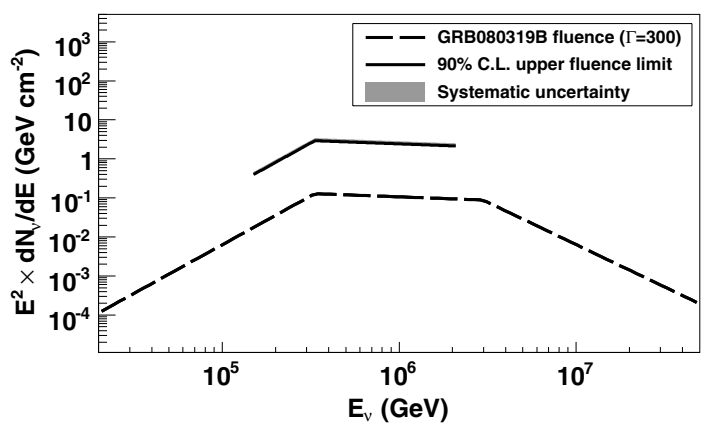

(b)

Fig. 6. (a) Integral limits at $90 \%$ C.L. on several $\nu_{\mu}+\bar{\nu}_{\mu}$ flux models (details in text). The energy ranges indicated contain $90 \%$ of the expected flux. The flux limits are for the full sky $(4 \pi \mathrm{sr})$, although only bursts from the Northern Hemisphere were used in the analysis. (b) Predicted $\nu_{\mu}+\bar{\nu}_{\mu}$ flux from for GRB 080319B using the Waxman-Bahcall model with observed burst parameters and assuming $\Gamma_{\text {jet }}=300$, compared to the $90 \%$ C.L. upper limit.

Nevertheless, the neutrino event rates are low (typically $\ll 1$ ) for individual bursts, so the searches are normally conducted by 'stacking' all known bursts and looking for neutrinos in conjunction with the ensemble as a whole.

The results of searches for emission from 419 GRBs observed in the Northern Hemisphere during stable AMANDA operations between 1997 and $2003^{45}$ is shown in Fig. 6(a). Three theoretical models are shown: the Waxman-Bahcall ${ }^{46}$ (divided by 2 to account for neutrino oscillations) and Murase-Nagataki ${ }^{47}$ calculations based on the assumption that GRBs are the sources of the ultrahigh-energy cosmic rays, and the "supranova" model of Razzaque et al. ${ }^{48}$ under the assumption that all GRBs are preceded by supernovae which produce ideal circumburst environments for neutrino production. For the latter two models, only the flux from long-duration GRBs is included; both long and short are included for the Waxman-Bahcall model.

Because limits are placed on the integrated flux predicted by the models, our constraints are given in terms of "model rejection factors" (MRFs), essentially the scaling factor at which the model would be just ruled out at $90 \%$ confidence. $^{49}$ MRFs less than 1 indicate that the model could be scaled down and still be excluded; i.e. that the model is excluded at the stated confidence level. The MRFs for the three models are 1.36 for the Waxman-Bahcall model, 0.92 for the MuraseNagataki parameter set A, and 0.45 for the supranova model under the assumptions mentioned above. A comparable search using IceCube observations is in progress.

A dedicated search was also conducted for neutrinos from the "naked-eye" GRB 080319B. ${ }^{50}$ Although 40 strings were in the ice at the time of this burst, the 18 new strings deployed in the 2007-08 austral summer had not yet been commissioned. More unfortunately, the burst occured during a maintenance period related to the transition to 40 -string operations, with only nine strings were taking data. 


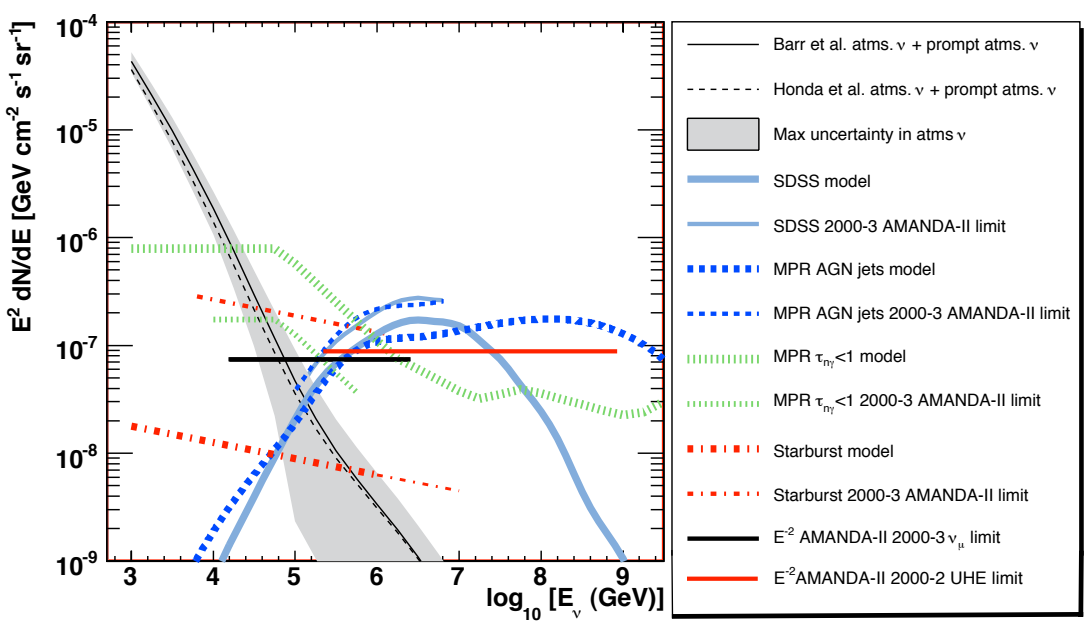

Fig. 7. AMANDA diffuse and ultrahigh-energy (UHE) integral limits on hypothetical $\nu_{\mu}+\bar{\nu}_{\mu}$ fluxes, compared to several diffuse flux predictions (thick lines, details in text) and the limits on those predictions (thin lines). The energy range for each limit is that within which $90 \%$ of detected neutrino events would fall.

Nonetheless, the extreme nature of the burst leads to a non-negligible predicted event rate in the 9-string detector. The limit placed on the Waxman-Bahcall flux, with parameters chosen according to those measured for GRB 080319B, is shown in Fig. 6(b) for an assumed jet Lorentz factor $\Gamma_{\text {jet }}=300$. For this choice of $\Gamma_{\text {jet }}$, 0.12 muon events are predicted, leading to an MRF of 22.7. Higher values of $\Gamma_{\text {jet }}$ lead to lower predicted event rates. Although the number of events expected for the 9-string configuration then operational is low, a similar burst observed with the full IceCube detector would be expected to produce $\mathcal{O}(1)$ event.

\subsection{Diffuse fluxes}

IceCube could also detect diffuse fluxes of high energy neutrinos, for example from a class of sources too dim to resolve individually. At relatively low energies such a flux would be indistinguishable from the atmospheric neutrino flux, but the soft spectrum of the conventional atmospheric neutrinos means that a diffuse astrophysical flux could be detected as a harder spectral component of the total diffuse flux.

Two independent searches for diffuse fluxes were undertaken using AMANDA data. The first ${ }^{30}$ was closely related to the standard muon neutrino analysis used to search for point sources of neutrinos, with cuts optimized for higher energy neutrinos and with a cut placed on the reconstructed energy of the neutrino candidate events. This search used data from 2000-2003, and the results are shown in Fig. 7. As a benchmark, the limit placed on a hypothetical diffuse $\nu_{\mu}$ flux $d N / d E=\Phi_{0} E^{-2}$ at $90 \%$ C.L. is $\Phi_{0} \geq 7.4 \times 10^{-8} \mathrm{GeV} \mathrm{cm}^{-2} \mathrm{~s}^{-1} \mathrm{sr}^{-1}$. For such a flux, $90 \%$ of the signal neutrinos would have had energies between $16 \mathrm{TeV}$ and $2.5 \mathrm{PeV}$, which are 
Table 2. Limits on several theoretical models of diffuse muon neutrino fluxes from the two analyses. The number of events that would have been detected $n_{\text {sig }}$ and the "model rejection factor" (MRF), the ratio of the upper limit to the predicted flux, are shown. MRFs less than 1 indicate that the model is excluded at the $90 \%$ C.L.

\begin{tabular}{|c|c|c|c|c|c|}
\hline Source & $n_{\text {sig }}^{\mathrm{HE}}$ & $\mathrm{MRF}^{\mathrm{HE}}$ & $n_{\mathrm{sig}}^{\mathrm{UHE}}$ & $\mathrm{MRF}^{\mathrm{UHE}}$ & Model \\
\hline \multirow[t]{6}{*}{ Active Galactic Nuclei } & 1.7 & 1.6 & 1.8 & 2.9 & Stecker ${ }^{52}$ \\
\hline & 1.4 & 2.0 & 5.9 & 0.9 & $\mathrm{MPR}^{15}$ \\
\hline & & & 8.8 & 0.6 & Halzen \& $\mathrm{Zas}^{3}$ \\
\hline & & & 20.6 & 0.3 & Protheroe $^{56}$ \\
\hline & & & 0.3 & 18.0 & Mannheim $^{57}$ RL A \\
\hline & & & 4.5 & 1.2 & Mannheim $^{57}$ RL B \\
\hline Starburst Galaxies & 1.1 & 21.1 & & & Loeb \& Waxman 58 \\
\hline \multirow{4}{*}{ Prompt Atmospheric $\nu_{\mu}$} & 0.4 & 60.3 & & & $\mathrm{MRS} \mathrm{GBW}^{28}$ \\
\hline & 4.7 & 5.2 & & & Naumov RQPM ${ }^{59}$ \\
\hline & 16.1 & 1.5 & & & Zas et al. ${ }^{60}$ Charm C \\
\hline & 26.2 & 0.95 & & & Zas et al. ${ }^{60}$ Charm D \\
\hline
\end{tabular}

the bounds of limit shown in Fig. 7. The limit is placed on the integrated flux, and so cannot be directly compared to specific theoretical models; instead, the predicted signal for each model must be simulated to find the model rejection factor (MRF). Limits on several specific models are also shown (as thin lines parallel to the predicted fluxes) in Fig. 7, and appear in Table 2. In addition to astrophysical models, a flux corresponding to the upper bound on generic optically thin $\left(\tau_{n \gamma}<1\right)$ pion photoproduction sites ${ }^{15}$ was tested. The upper limit from the analysis is a factor of 0.22 of the MPR bound over the region from $10 \mathrm{TeV}$ to $630 \mathrm{TeV}$.

A second analysis based on data from 2000-2002 exploited idiosyncracies of the hardware response to the extremely bright events produced by ultrahigh energy (UHE) neutrinos, such as afterpulsing in the PMTs, to extend the range of the detector to much higher energies. ${ }^{51}$ The limit for the $E^{-2}$ benchmark $\nu_{\mu}$ flux is $9.0 \times 10^{-8} \mathrm{GeV} \mathrm{cm}^{-2} \mathrm{~s}^{-1} \mathrm{sr}^{-1}$ at $90 \%$ C.L. (assuming a $1: 1: 1$ flavor ratio), over a range from $2 \times 10^{5} \mathrm{GeV}$ to $10^{9} \mathrm{GeV}$, as shown in Fig. 7. It should be noted that the analysis was sensitive to all flavors of neutrinos; for a $E^{-2}$ spectrum with flavor equality, the flavor ratio of the detected events would have been approximately $2: 2: 1$. Limits on specific theoretical models need to be calculated separately for this analysis and are omitted from Fig. 7 for clarity but are shown in Table 2.

\section{Dark Matter}

Large volume neutrino telescopes such as IceCube can search for indirect evidence of dark matter, such as supersymmetric neutralinos, that could accumulate in the gravitational wells of the Earth and Sun and annihilate to produce neutrinos. These searches are complementary to those conducted by direct detection experiments, because the latter generally rely on coherent scattering of the WIMP from the ensemble of nucleons in heavy nuclei. Direct detection experiments thus constrain 


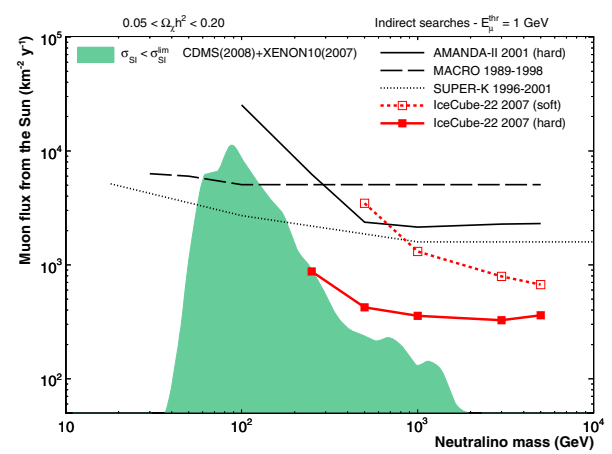

(a)

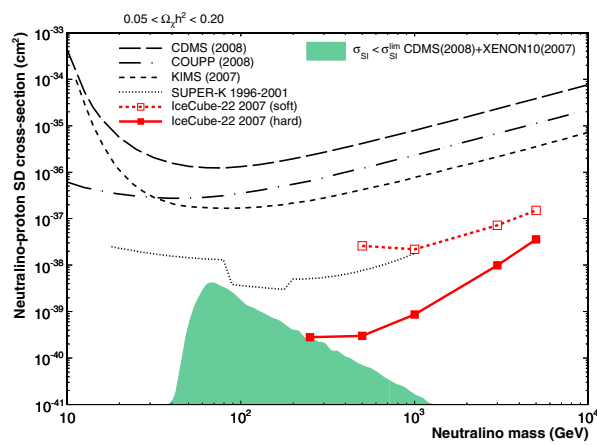

(b)

Fig. 8. (a) Limits on the flux of neutrino-induced muons due to neutralino annihilation in the Sun, normalized to a $1 \mathrm{GeV}$ muon threshold with assumed hard $\left(\chi \chi \rightarrow W^{+} W^{-}\right)$and soft $(b \bar{b})$ neutrino spectra. The shaded region indicated the allowed MSSM parameter space. (b) Limits on the spin-dependent neutralino-proton cross section inferred based on the muon limits.

primarily the spin-independent neutralino-nucleon scattering cross section $\sigma_{\mathrm{SI}}$. Neutralino capture in the Sun, which is made primarily of light nuclei, allows us to probe models where the coupling is primarily spin-dependent. ${ }^{53}$ It should also be noted that direct and indirect searches probe different epochs of the history of the solar system and different parts of the WIMP velocity distribution.

Figure $8(\mathrm{a})$ shows the limit on the flux of muons produced by neutrinos generated in neutralino annihilations in the sun with the 22 -string IceCube detector, ${ }^{54}$ compared to those set by other neutrino detectors. ${ }^{64,63,65}$ This analysis used 104 days of live time from June to September 2007, while the Sun was below the horizon as viewed from the South Pole. This limit includes a full simulation of WIMP annihilation in the Sun and neutrino transport through the Sun and Earth ${ }^{55}$ as well as of the IceCube detector, and for comparison between experiments the flux is quoted above an arbitrary threshold of $1 \mathrm{GeV}$. In Fig. 8(b), the limit on the WIMP annihilation rate shown in Fig. 8(a) is converted to a limit on the spin-dependent scattering cross section $\sigma_{\mathrm{SD}}$, using the conservative assumption that $\sigma_{\mathrm{SI}}=0$. The limits on $\sigma_{\mathrm{SD}}$ from direct detection experiments ${ }^{62,61,66,67}$ and Super-K $\mathrm{K}^{63}$ are also shown. In both figures, the shaded area represents the muon flux or $\sigma_{\mathrm{SD}}$ predicted by MSSM models not excluded by direct detection experiments on the basis of their limits on $\sigma_{\mathrm{SI}}$. It should be noted that most of this model space includes relatively weak spin-independent coupling, so that very significant improvements (more than 3 orders of magnitude) in sensitivity would be required for direct detection experiments to significantly constrain the remaining models. An analysis of the sensitivity to other types of dark matter, such as Kaluza-Klein particles, is in progress.

The future reach of dark matter searches will be greatly enhanced by the completion of IceCube and the addition of the Deep Core array, which will substantially improve IceCube's sensitivity to neutralino masses below a few hundred GeV. 


\section{Outlook}

Construction of the IceCube detector is proceeding very well, with completion of the array expected in 2011. IceCube will be augmented with the Deep Core array, to be completed in 2010, which will significantly extend its capabilities at energies as low as $10 \mathrm{GeV}$. Initial results from the partially built detector, including only one quarter of the final array, are already providing sensitivities beyond those of the complete seven-year AMANDA-II data set, and this sensitivity will expand rapidly as construction progresses. Within a few years the sensitivity of IceCube will be sufficient to detect astrophysical neutrino fluxes at the levels expected on theoretical grounds to be produced by the sources of the cosmic rays. In addition, IceCube and Deep Core will permit indirect searches for dark matter well beyond existing limits, and studies of atmospheric neutrinos with unprecedented statistics.

\section{Acknowledgments}

The author is grateful for the hospitality of the Aspen Institute and for valuable discussions with many participants in the 2009 neutrino physics workshop during the preparation of this review. This work was supported by NSF grant PHY-0554868.

\section{References}

1. A. M. Hillas, arXiv:astro-ph/0607109.

2. P. Blasi, Mod. Phys. Lett. A 20, 3055 (2005).

3. F. Halzen and E. Zas, Astrophys. J. 488, 669 (1997).

4. V. S. Berezinsky, C. Castagnoli and P. Galeotti, Nuovo Cimento C 8, 185 (1985) [Addendum-ibid. 8, 602 (1985)].

5. F. W. Stecker, O. C. de Jager and M. H. Salamon, Astrophys. J. 390, L49 (1992).

6. J. P. Rachen and P. Meszaros, Phys. Rev. D 58, 123005 (1998).

7. T. Kashti and E. Waxman, Phys. Rev. Lett. 95, 181101 (2005).

8. L. A. Anchordoqui et al., Phys. Lett. B 593, 42 (2004).

9. S. Razzaque, P. Meszaros and E. Waxman, Phys. Rev. D 73, 103005 (2006).

10. J. F. Beacom, N. F. Bell, D. Hooper, S. Pakvasa and T. J. Weiler, Phys. Rev. D 68, 093005 (2003) [Erratum-ibid. 72, 019901 (2005)].

11. F. Halzen and D. Hooper, Rep. Prog. Phys. 65, 1025 (2002).

12. M. Boettcher, Astrophys. Space Sci. 309, 95 (2007).

13. P. Meszaros, AIP Conf. Proc. 1040, 150 (2008).

14. E. Waxman and J. N. Bahcall, Phys. Rev. D 59, 023002 (1999).

15. K. Mannheim, R. J. Protheroe and J. P. Rachen, Phys. Rev. D 63, 023003 (2001).

16. E. Migneco, J. Phys. Conf. Ser. 136, 022048 (2008).

17. IceCube Collab. (R. Abbasi et al.), Nucl. Instrum. Meth. A 601, 294 (2009).

18. IceCube Collab. (T. Stanev et al.), arXiv:0903.0576 [astro-ph.HE].

19. IceCube Collab. (R. Abbasi et al.), arXiv:0810.2034 [astro-ph].

20. R. Gandhi, C. Quigg, M. H. Reno and I. Sarcevic, Astropart. Phys. 5, 81 (1996).

21. T. K. Gaisser, Cosmic Rays and Particle Physics (Cambridge Univ. Press, 1992).

22. J. G. Learned and S. Pakvasa, Astropart. Phys. 3, 267 (1995).

23. T. DeYoung, S. Razzaque and D. F. Cowen, Astropart. Phys. 27, 238 (2007).

24. I. Albuquerque, G. Burdman and Z. Chacko, Phys. Rev. Lett. 92, 221802 (2004). 
25. L. V. Volkova, Sov. J. Nucl. Phys. 31, 784 (1980) [Yad. Fiz. 31, 1510 (1980)].

26. J. G. Learned and K. Mannheim, Ann. Rev. Nucl. Part. Sci. 50, 679 (2000).

27. M. Thunman, G. Ingelman and P. Gondolo, Astropart. Phys. 5, 309 (1996).

28. A. D. Martin, M. G. Ryskin and A. M. Stasto, Acta Phys. Pol. B 34, 3273 (2003).

29. R. Enberg, M. H. Reno and I. Sarcevic, Phys. Rev. D 78, 043005 (2008).

30. IceCube Collab. (A. Achterberg et al.), Phys. Rev. D 76, 042008 (2007) [Erratum-ibid. 77, 089904 (2008)].

31. G. D. Barr, T. K. Gaisser, S. Robbins and T. Stanev, Phys. Rev. D 74, 094009 (2006).

32. G. D. Barr et al., Phys. Rev. D 70, 023006 (2004).

33. M. Honda et al., Phys. Rev. D 75, 043006 (2007).

34. IceCube Collab. (R. Abbasi et al.), Phys. Rev. D 79, 102005 (2009).

35. Super-Kamiokande Collab. (Y. Ashie et al.), Phys. Rev. D 71, 112005 (2005).

36. M. C. Gonzalez-Garcia, M. Maltoni and J. Rojo, JHEP 0610, 075 (2006).

37. O. Mena, I. Mocioiu and S. Razzaque, Phys. Rev. D 78, 093003 (2008).

38. IceCube Collab. (R. Abbasi et al.), Phys. Rev. D 79, 062001 (2009).

39. AMANDA Collab. (J. Ahrens et al.), Nucl. Inst. Meth. A 524, 169 (2004).

40. Milagro Collab. (A. A. Abdo et al.), Astrophys. J. 664, L91 (2007).

41. Milagro Collab. (A. A. Abdo et al.), arXiv:0904.1018 [astro-ph.HE].

42. Fermi LAT Collab. (A. A. Abdo et al.), arXiv:0902.1340 [astro-ph.HE].

43. IceCube Collab. (R. Abbasi et al.), arXiv:0905.2253 [astro-ph.HE].

44. ANTARES Collab. (J. A. Aguilar Sanchez), arXiv:0710.0252 [astro-ph].

45. The IceCube Collab. and the IPN Collab. (A. Achterberg et al. and K. Hurley et al.), arXiv:0705.1186 [astro-ph].

46. E. Waxman, Nucl. Phys. Proc. Suppl. 118, 353 (2003).

47. K. Murase and S. Nagataki, Phys. Rev. D 73, 063002 (2006).

48. S. Razzaque, P. Meszaros and E. Waxman, Phys. Rev. Lett. 90, 241103 (2003).

49. G. C. Hill and K. Rawlins, Astropart. Phys. 19, 393 (2003).

50. IceCube Collab. (R. Abbasi et al.), arXiv:0902.0131 [astro-ph.HE].

51. IceCube Collab. (M. Ackermann et al.), Astrophys. J. 675, 1014 (2008).

52. F. W. Stecker, Phys. Rev. D 72, 107301 (2005).

53. F. Halzen and D. Hooper, Phys. Rev. D 73, 123507 (2006).

54. IceCube Collab. (R. Abbasi et al.), arXiv:0902.2460 [astro-ph.CO].

55. M. Blennow, J. Edsjo and T. Ohlsson, J. Cosmol. Astropart. Phys. 0801, 021 (2008).

56. R. J. Protheroe, arXiv:astro-ph/9607165.

57. K. Mannheim, Astropart. Phys. 3, 295 (1995).

58. A. Loeb and E. Waxman, J. Cosmol. Astropart. Phys. 0605, 003 (2006).

59. V. A. Naumov, arXiv:hep-ph/0201310.

60. E. Zas, F. Halzen and R. A. Vazquez, Astropart. Phys. 1, 297 (1993).

61. CDMS Collab. (Z. Ahmed et al.), Phys. Rev. Lett. 102, 011301 (2009).

62. XENON Collab. (J. Angle et al.), Phys. Rev. Lett. 100, 021303 (2008).

63. Super-Kamiokande Collab. (S. Desai et al.), Phys. Rev. D 70, 083523 (2004) [Erratumibid. 70, 109901 (2004)].

64. MACRO Collab. (M. Ambrosio et al.), Phys. Rev. D 60, 082002 (1999).

65. AMANDA Collab. (M. Ackermann et al.), Astropart. Phys. 24, 459 (2006).

66. KIMS Collab. (H. S. Lee et al.), Phys. Rev. Lett. 99, 091301 (2007).

67. COUPP Collab. (E. Behnke et al.), Science 319, 933 (2008). 